\title{
Computer program to generate operant schedules
}

\author{
ALLEN H. WOLACH \\ Illinois Institute of Technology, Chicago, Illinois \\ and \\ MAUREEN A. MCHALE \\ Northwestern State University of Louisiana, Natchitoches, Louisiana
}

\begin{abstract}
A computer program for programming schedules of reinforcement is described. Students can use the program to experience schedules of reinforcement that are typically used with nonhuman subjects. A cumulative recording of a student's responding can be shown on the screen and/or printed with the computer's printer. The program can also be used to program operant schedules for animal subjects. The program was tested with human subjects experiencing fixed ratio, variable ratio, fixed interval, and variable interval schedules. Performance for human subjects on a given schedule was similar to performance for nonhuman subjects on the same schedule.
\end{abstract}

A computer program for controlling contingencies of reinforcement on operant schedules has been developed. The program can be used in two ways. First, the program can be used so that students can experience contingencies of reinforcement that are typically experienced by nonhuman subjects. Second, the computer program and interface box described below can be used for research with animal subjects to reduce the cost of performing research. The program generates cumulative recordings that can be printed with a printer that is attached to the computer. When the program is used with human subjects, no apparatus other than a computer and printer is required.

The present computer program provides a wide variety of schedules of reinforcement. These schedules include most of the schedules reported in Ferster and Skinner (1957).

When the program is used to allow students to experience schedules of reinforcement, on-screen displays of schedule parameters, cumulative responses, and cumulative reinforcements can be turned off. A student can use the left mouse button (or the "A" key on the keyboard) to simulate a manipulandum in an operant chamber. The student can respond to a schedule in an attempt to identify contingencies of reinforcement that are in effect. When the schedule of reinforcement determines that a response should be reinforced, the word "Reinforced" flashes on the screen after the student makes the response. A red or blue disk appears on the screen when a discriminative stimulus is required. For example, a red disk appears for the first component of a chained schedule, followed by a blue disk for the second component. After the student is finished with the operant session, a cumulative recording of the student's responses can be shown on the screen or printed with a printer.

The approach to presentation of operant schedules that is used in the present program is different from the ap-

Correspondence concerning this article should be addressed to A. H. Wolach, Institute of Psychology, Illinois Institute of Technology, Chicago, IL 60616-3793 (e-mail: wolach@iit.edu). proach that is used in computer simulations for operant laboratories. Consider Sniffy the Virtual Rat (2000). This program allows a student to shape the responses of a virtual rat. Such a program has the advantage of allowing the student to shape the responses of the virtual rat in a situation that mimics a laboratory study with a real rat. The problem with this approach is that the program is sensitive only to the rules of shaping that are programmed into the simulation. An investigator who is good at shaping subjects can learn to successfully shape the responses of real subjects by reinforcing approximations of behavior that are not reinforced by other investigators. These approximations are not programmed into a simulation.

Early in training, Sniffy is very sensitive to reinforcement after standing on his hind legs. However, Sniffy is not programmed to approach the lever from diverse resting positions. In addition, the angle from which Sniffy is viewed does not make it possible to determine how far he is from the lever. Students who attempt to use distance from the lever and angle of approach (as opposed to rearing up) as cues for delivering reinforcement will not successfully shape Sniffy's responses. A simulation such as Sniffy the Virtual Rat (2000) is a rational, as opposed to an empiri$\mathrm{cal}$, demonstration of shaping. Students who reshape Sniffy cannot get a feel for the diverse ways in which real rats respond to shaping.

The present program does not have the problem of limited sensitivity, because the student is a real subject. If the student experiences a schedule for a number of days, he or she can learn about schedule control and the ability to describe contingencies of reinforcement for the schedule.

\section{PROGRAM OPERATION}

\section{Menu Bar}

The program starts with a menu bar that provides the following options: File, Schedules, System Setup, and $\underline{H}$ elp. If the user chooses $\underline{H}$ elp from the menu bar, he or 


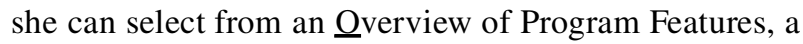
menu system that contains a description of all the schedules of reinforcement that are programmed by the system and directions for interfacing a microcomputer with an operant chamber. The directions include methods for connecting the contacts from an operant chamber microswitch to a mouse or to a keyboard and for making an interface box that will allow the computer to control a feeder.

\section{System Setup}

The program can be used in two modes, $\underline{\text { On Screen }}$ Demonstration, and Manipulandum and Feeder Attached

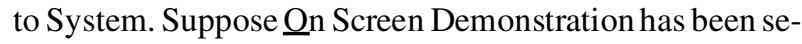
lected from the System Setup menu item on the menu bar before $\underline{S}$ chedules is selected from the menu bar. Further suppose that Do Not Show Responses and Schedule Information On Screen is selected from the System Setup menu.

\section{Schedules Option}

An instructor or teaching assistant can choose the options described above for each computer in a student laboratory. The instructor can select a schedule from the

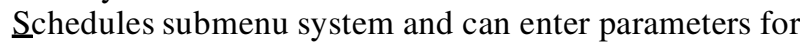
the schedule (e.g., the number of presses required for reinforcement on a fixed ratio [FR] schedule). The instructor can choose options and a specific schedule while the student is not viewing the monitor screen. Then the student can be seated facing the monitor screen to left-click the mouse on the Operate Manipulandum button or hold the Alt key down and press the letter "A" on the keyboard. Left-clicking the Operate Manipulandumbutton or pressing the letter " $\mathrm{A}$ " is analogous to the response a subject makes to a manipulandum in an operant chamber. Later, the student or instructor can print the cumulative recording and compare it with cumulative recordings from nonhuman subjects.
The mouse is used to select a category for a schedule of reinforcement from the $\underline{S}$ chedules option of the menu bar. The categories are Magazine Training, Nonintermittent Schedules, Intermittent Schedules, and Differential Reinforcement (see, e.g., Ferster \& Skinner, 1957). Magazine Training includes options with and without the lever active during magazine training (e.g., Ferster \& Skinner, 1957). Nonintermittent Schedules include continuous reinforcement and extinction (e.g., Ferster \& Skinner, 1957). Intermittent Schedules include FR, fixed interval (FI), variable ratio (VR), variable interval (VI), alternative, conjunctive, interlocking, tandem (FI FR, FI VR, FI VI, FI FI, VI FR, VI FI, FR FI, FR VI, VR FI), chained (same options as tandem), adjusting (by pause after reinforcement, by time to complete ratio, by interval, and by the time a subject takes to respond after reinforcement is delivered), multiple (two from among FR, VR, FI, and VI), mixed (same options as multiple), and concurrent (same options as multiple) schedules (e.g., Ferster \& Skinner, 1957). Differential reinforcement schedules include low and high rates (e.g., Ferster \& Skinner, 1957; Reynolds, 1975).

All the schedules (except continuous reinforcement and extinction) allow the user to enter parameters for the schedule (e.g., the FI for a FI schedule). The user enters the parameters with the mouse and/or the keyboard. Note that the user can receive help about the contingencies for the schedule by clicking the command button labeled Show Description of Schedule at the bottom of the screen or by

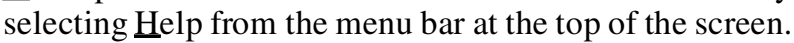
The screen for entering parameters for a concurrent schedule is shown in Figure 1.

If Show Responses and Schedule Information on Screen is selected from the System Setup menu before a schedule is selected, the name of the schedule and the parameters for the schedule appear on the screen. A running total of number of responses and number of reinforced responses also appears on the screen.

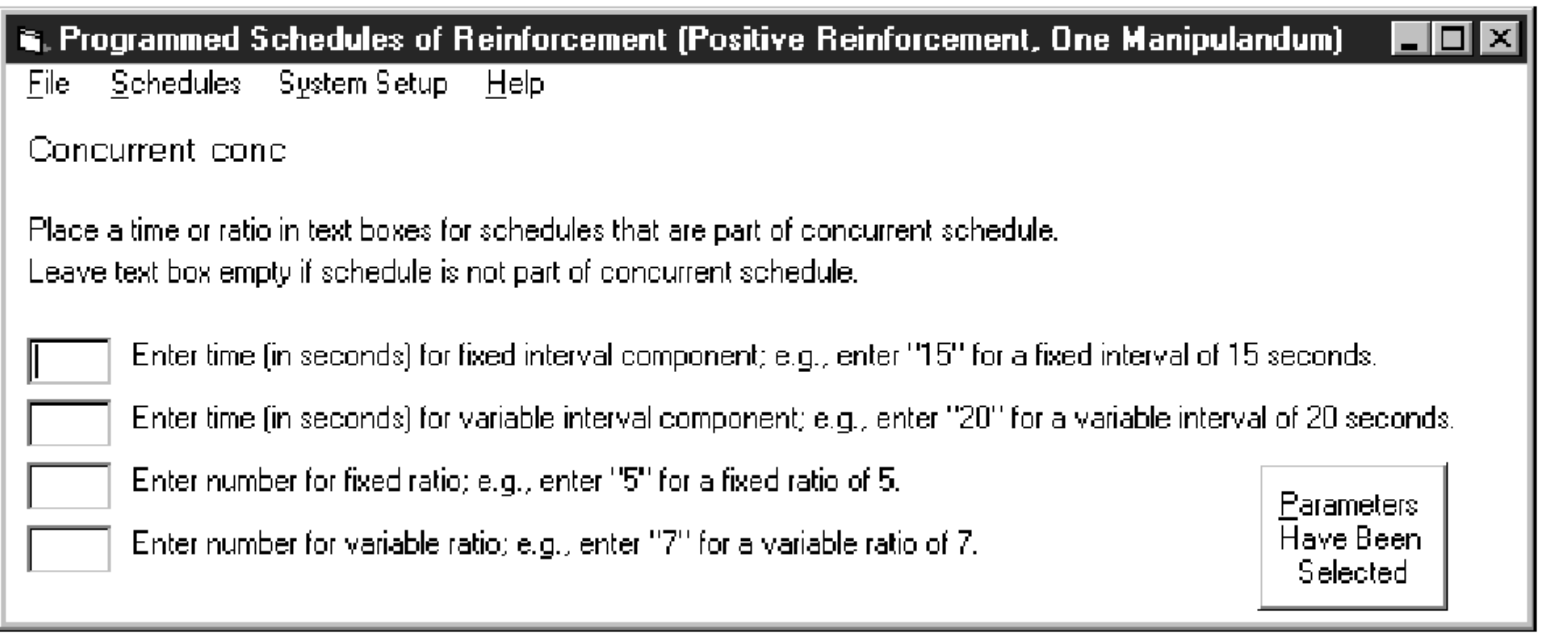

Figure 1. Screen for entering parameters for a concurrent schedule. 


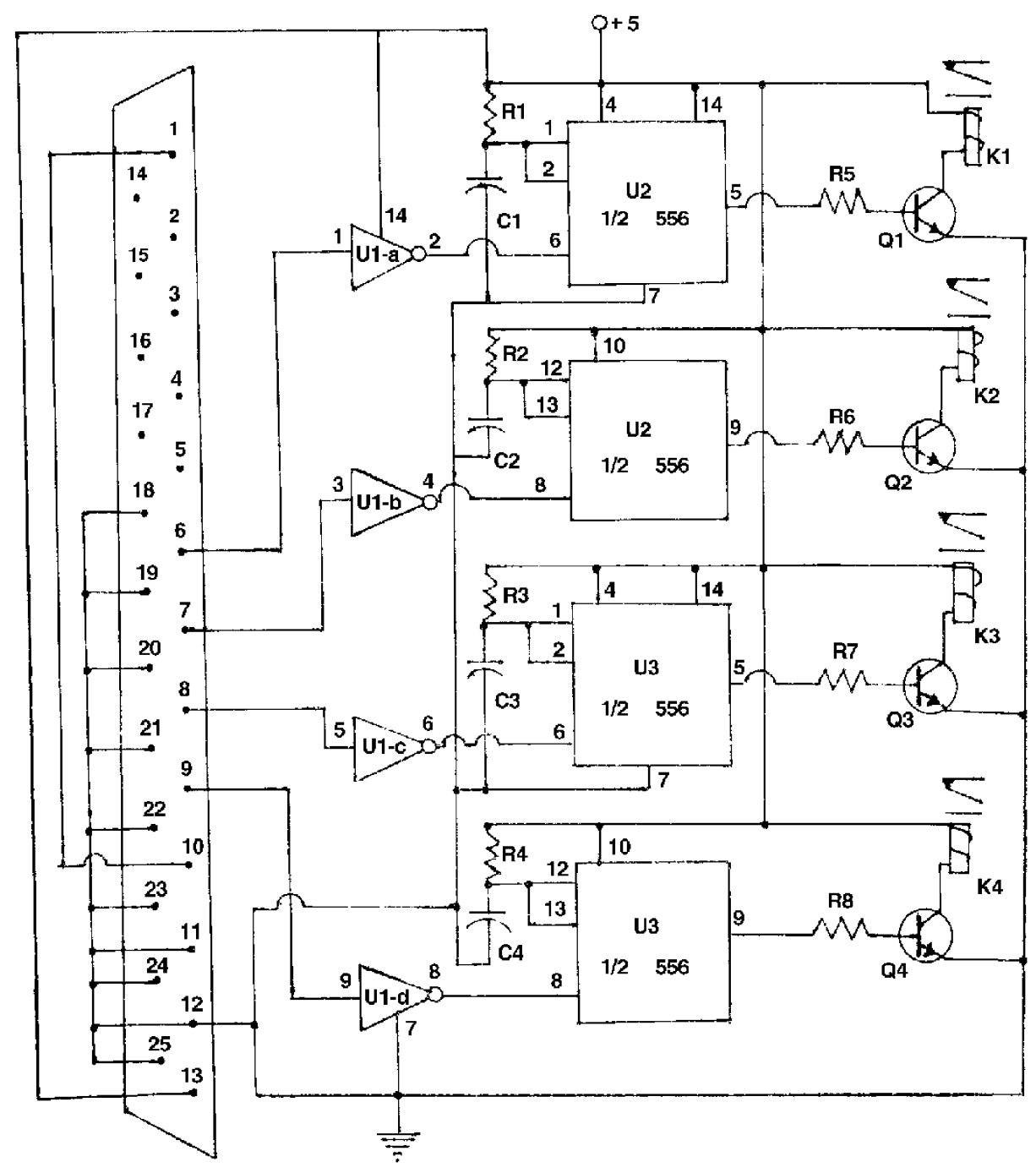

Figure 2. Schematic diagram for the interface box that connects to parallel printer port and feeder. The connector at the left of the figure is a male DB25 connector. Integrated circuit U1 (74LS04) contains four inverters. Integrated circuits $U 2$ and $U 3$ contain two 556 timers. Resistors $R 1$ through $R 8$ are 10,000 ohms. Capacitors $C 1$ through $C 4$ are $47 \mathrm{mfd}$. Transistors Q1 through Q4 are switching transistors (MP52222A or MP53904). Relays K1 through K4 are 5-V relays (SPST) capable of switching 1 amp (Radio Shack 225-232) or 2 amps (Radio Shack 225-243).

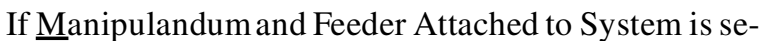
lected from the System Setup menu, the schedules described above can be used to operate an interface box that is attached to an operant chamber. The interface box controls relays used to activate a feeder and turns lights for discriminative stimuli on and off.

Help for a schedule can be selected from the menu bar at the top of the screen. Help is inactivated when an instructor clicks on Do Not Show Responses and Schedule Information on Screen. When Help is inactivated, a student cannot use it to identify the schedule he is experiencing. Help for the schedule in question describes the schedule and also describes constraints on the present implementation of the schedule. When necessary, $\underline{\text { Help for }}$ the schedule lists limitations on the parameters that can be entered.

When the session is completed (student or animal subject receives the requisite number of reinforcements or makes the requisite number of responses or an appropriate session time elapses), End Session command button is clicked. The Operate Manipulandum button and the manipulandum (for nonhuman subjects) are inactivated, and the elapsed time for the session clock stops when the End Session command button is clicked.

After the End Session command button is clicked, the investigator can click on the button labeled Save Cumulative Record to save the data for the cumulative recording to a file. When the option to $\underline{\text { Save Cumulative Record is }}$ 


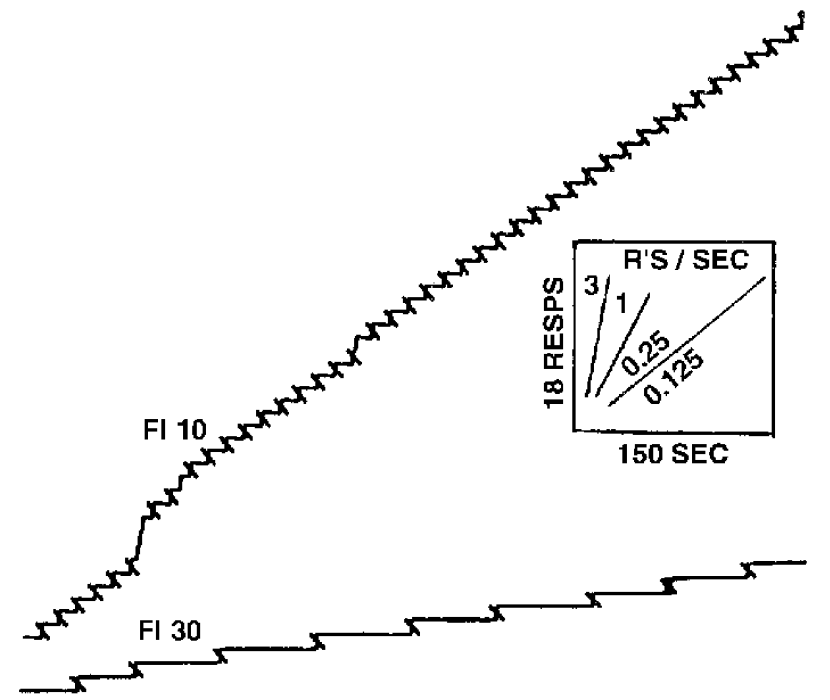

Figure 3. Fixed interval (FI) performance on Day 10 for subjects who experienced FI 10 and FI 30 with no printed description of the schedule.

chosen, a standard Windows dialog box for saving the file for the cumulative recording appears on the screen. The investigator enters a name for the file that is to contain information for the cumulative record. The user is also given the option of entering information (text) that will appear at the top of the cumulative recording. For example, the user can enter the subject number and the session number.

\section{File Menu on Menu Bar}

The File menu has options to Print Cumulative Recording and View Cumulative Recording on Screen.

\section{System Setup Menu on Menu Bar}

Some of the options on the System Setup menu have been discussed above. The System Setup menu shows the following options:

\section{On Screen Demonstration}

Manipulandum and Feeder Attached to System

Show Responses and Schedule Information on Screen

Do Not Show Responses and Schedule Information on Screen

Choose Printer Font

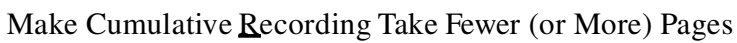

12 (Shortest Cumulative Recording)

$\underline{25}$ (Second Shortest Cumulative Recording)

$\underline{50}$ (Typical X-Y Ratio for Cumulative Recording)

$1 \underline{0} 0$ (Second Longest Cumulative Recording)

150 (Longest Cumulative Recording)

[ Print Quality of Cumulative Recording
Draft Quality

Typical

Letter Quality

Conventional cumulative recorders move paper at a fixed speed. If the speed of the paper drive is increased, the cumulative recording requires more paper. Options under Make Cumulative Recording Take Fewer (or More) Pages are used to simulate several paper speeds. The numbers $(12,25,50,100$, and 150$)$ relate to the $X-Y$ ratio for the cumulative recording, multiplied by 100 . A value of 50 produces a cumulative recording that looks like the cumulative recording from a typical mechanical cumulative recorder.

Options under Print Quality of Cumulative Recording are used to increase or decrease the thickness and regularity of the cumulative recording. Draft Quality produces a thin cumulative recording with some variations in line thickness. However, Draft Quality cumulative recordings print more rapidly, as compared with Letter Quality cumulative recordings. The Print Quality of Cumulative Recording option modifies print quality for dot matrix, laser, and ink jet printers.

\section{Interfacing Box for Operant Chamber}

The Help system for the program and the manual (file Manual on the second installation disk) include detailed directions for interfacing the computer program to an operant chamber. Figure 2 shows the schematic diagram for the interface box that is attached to the printer port of the computer. Detailed directions for making the interface box are included in the manual. See Programming Schedules of Reinforcement with Integrated Circuits (Wolach, 1979) for a primer on integrated circuits.

\section{REINFORCEMENT SCHEDULES WITH HUMAN SUBJECTS}

The program was tested with human subjects experiencing FR 10, FR 20, FR 40, VR 10, VR 20, FR 80, FI 10, FI 30, FI 60, VI 10, and VI 30 schedules. Some research suggests that subjects who receive no instructions about their schedule of reinforcement fail to acquire response patterns that have been obtained with nonhuman subjects on the same schedule (e.g., Baron, Kaufman, \& Stauber, 1969; Turner \& Solomon, 1962). When human subjects experience instructions about a potential schedule, they often produce response patterns that are close to the response patterns for nonhuman subjects (e.g., Ayllon \& Azrin, 1961; Baron \& Kaufman, 1966). Half the subjects on FR, VR, FI, and VI schedules in the present study were given a description of their schedule before starting 10 days of training ( 9 min per day). A prior description of the schedule versus no prior description of the schedule did not differentially affect performance on the schedules.

After 10 days of training, cumulative recordings for human subjects on FR, VR, FI, and VI schedules were much like cumulative recordings for nonhuman subjects. 
As fixed intervals increase, pigeons and rats decrease rate of responding during FIs (Holland, 1958). Holland found that human subjects also decreased rate of responding as FIs increased. Other investigators (Blair, 1958; Weiner, 1962) have found comparable high rates of responding independent of the FI for human subjects (Blair, 1958; Weiner, 1962). The present study, like Holland's study, showed lower response rates as FIs increased. Humans, as compared with rats and pigeons, took longer pauses on FI schedules. Figure 3 shows cumulative recordings generated by the program (Day 10) for subjects on FI 10 and FI 30 schedules. After 10 days of training, some human subjects waited the entire FI before responding for reinforcement.

The four basic schedules of reinforcement can gain control over the behavior of human subjects experiencing short session times and only 10 days of training. The method for presenting schedules to human subjects with the present program produced performance that was very similar to performance for nonhuman subjects.

A detailed description of the study that was performed to test the schedules of reinforcement on human subjects is in the file Study.RTF on the second distribution disk for the computer program.

\section{AVAILABILITY AND EQUIPMENT REQUIREMENTS}

If two formatted 3.5-in. high-density disks are included with the request, the program is available free of charge. Alternatively, \$2 may be enclosed with the request to cover the cost of the disks. The program was written in $\mathrm{Vi}$ sual Basic, Version 6, and runs on IBM-compatible computers that have Windows 95, 98, Me, NT, or 2000 as an operating system. A standard Windows setup program is included on the disks. That is, the user places the first of the two disks in the floppy drive, selects Run from the Start menu, and types A:ISetup. The program requires less than $1 \mathrm{MB}$ of hard drive space. A printer is required for users who want to print cumulative recordings generated by the program. If the program is used to control an operant chamber, the parallel port of the computer can be used to connect the interface box that controls the feeder in the operant chamber. The user can switch cables from the printer and the interface box or can connect the cables to a switch box. A mouse or keyboard that is attached to the computer requires a slight modification to accept input from the lever in the operant chamber. The modification is described under Help and requires soldering wires to the two contacts of the "A" key of the keyboard.

\section{REFERENCES}

Ayllon, T., \& Azrin, N. H. (1961). Free-operant avoidance conditioning in human subjects. Journal of the Experimental Analysis of Behavior, 4, 275-276.

Baron, A., \& KAUfMan, A. (1966). Human, free-operant avoidance of "time out" from monetary reinforcement. Journal of the Experimental Analysis of Behavior, 9, 557-565

Baron, A., Kaufman, A., \& Stauber, K. A. (1969). Effects of instructions and reinforcement feedback on human operant behavior maintained by fixed interval reinforcement. Journal of the Experimental Analysis of Behavior, 12, 701-712.

BlaIR, W. C. (1958). Measurement of observing responses in human monitoring. Science, 128, 255-256.

Ferster, C. B., \& Skinner, B. F. (1957). Schedules of reinforcement. New York: Appleton-Century-Crofts.

Holland, J. G. (1958). Human vigilance. Science, 128, 61-63.

REYNOLDS, G. S. (1975). A primer of operant psychology (Rev. ed.). Glenview, IL: Scott, Foresman.

Sniffy the virtual rat [Computer software] (2000). Belmont, CA: Wadsworth/Thompson Learning.

Turner, L. H., \& Solomon, R. L. (1962). Human traumatic avoidance learning: Theory and experiments on the operant-respondent distinction and failures to learn. Psychological Monographs, 76, (40, Whole No. 559).

WeINer, H. (1962). Some effects of response cost upon human operant behavior. Journal of the Experimental Analysis of Behavior, 5, 201208

Wolach, A. H. (1979). Programming schedules of reinforcement with integrated circuits. Chicago: K.D.V.H.E.

(Manuscript received August 13, 2001; revision accepted for publication February 24, 2002.) 